\title{
Produção de painéis aglomerados da madeira de desrama de Acacia mangium
}

José Benedito Guimarães Junior ${ }^{1}$, Bruna Layara Messias Araújo², Olívia Pereira Lopes ${ }^{3}$, Rafael Farinassi Mendes ${ }^{3}$, Lourival Marin Mendes ${ }^{3}$

${ }^{1}$ Universidade Federal de Goiás, Rodovia BR-364, Setor Francisco Antônio, CEP 75801-615, Jataí, GO, Brasil

2Universidade Federal do Piauí, Campus Universitário Ministro Petrônio Portela, s/n, Iningá, CEP 64049-550, Teresina, PI, Brasil

3Universidade Federal de Lavras, Departamento de Ciências Florestais, CEP 37200-000, Lavras, MG, Brasil

"Autor correspondente:

jbguimaraesjr@hotmail.com

Termos para indexação:

Módulo de elasticidade

Módulo de ruptura

Resíduo florestal

Index terms:

Modulus of elasticity

Modulus of rupture

Wood waste

\section{Histórico do artigo:}

Recebido em 12/09/2012

Aprovado em 25/11/2013

Publicado em 31/12/2013

doi: 10.4336/2013.pfb.33.76.434
Resumo - Esse trabalho teve como objetivo a avaliar a qualidade de painéis aglomerados de madeira de desrama de Acacia mangium Willd, de forma comparativa com painéis de Pinus oocarpa e Eucalyptus grandis. A madeira de A. mangium foi obtida no sul do Estado do Piauí e as amostras de madeira de P. oocarpa e E. grandis foram coletadas em plantios na cidade de Lavras, MG. Para a produção dos painéis foi utilizado $8 \%$ de adesivo uréia-formaldeído e $1 \%$ de parafina. Os painéis foram prensados a $160{ }^{\circ} \mathrm{C}$, pressão de 3,92 MPa por 8 minutos. Considerando as propriedades físicas, os painéis de madeira de desrama de $A$. mangium e $P$. oocarpa apresentaram os melhores resultados, com destaque para a desrama de $A$. mangium, uma vez que apresentou valores de inchamento em espessura inferiores aos exigidos pela norma CS 236-66. De acordo com a mesma norma, apenas $E$. grandis apresentou resultados acima das exigências para o módulo de elasticidade e todos os materiais apresentaram desempenho superior às referências normatizadas para o módulo de ruptura. Para tração perpendicular destacaram-se os painéis produzidos com a madeira de desrama de A. mangium e $E$. grandis, com valores elevados para essa propriedade mecânica.

\section{Particleboard production from Acacia mangium pruning wood}

\begin{abstract}
The objective of this study was to assess the quality of particleboard of Acacia mangium Willd pruning wood, in comparison with Pinus oocarpa and Eucalyptus grandis panels. The wood of $A$. mangium was obtained in the southern region of Piaui State, and the wood samples of $P$. oocarpa and E. grandis were obtained in Lavras, State of Minas Gerais. To produce the panels, it was used $8 \%$ of urea-formaldehyde adhesive and $1 \%$ of paraffin. The panels were pressed at $160{ }^{\circ} \mathrm{C}$, pressure of $3.92 \mathrm{Mpa}$ for $8 \mathrm{~min}$. Considering the physical properties, the panels of wood pruning from $A$. mangium and from $P$. oocarpa showed the best results, especially from $A$. mangium that presented values of thickness swelling below those required by the standard CS 236-66. According to the same standard, only E. grandis panels showed results above requirements to the modulus of elasticity and all materials presented performance above the reference normalized for the modulus of rupture. To traction strain the panels produced with pruning wood of A. mangium and E. grandis, with higher values for this mechanical property.
\end{abstract}




\section{Introdução}

Os painéis aglomerados caracterizam-se pela transformação da madeira em pequenas partículas que, secas e misturadas com adesivos sintéticos termofixos e distribuídas aleatoriamente entre si, são prensadas sob calor e pressão, gerando um painel particulado (Moslemi, 1974; Tsoumis, 1991). De acordo com esse conceito, pode-se dizer que todo e qualquer material lignocelulósico tem potencial para ser utilizado como matéria-prima para a fabricação de chapas de partículas. Nesse sentido, confere a oportunidade de utilização de resíduos florestais e agroindustriais na sua produção (Guimarães Junior et al., 2011).

De acordo com Fagundes (2003), a utilização de resíduos gerados pela indústria florestal contribui para aumentar a sustentabilidade de utilização das florestas, de forma especial as plantadas, além de gerar benefícios sociais. Bufalino (2010) enfatiza ainda que essa prática é especialmente interessante em locais onde há escassez de madeira.

Atualmente, as madeiras de pinus e eucalipto constituem a base de matéria-prima para produção de painéis aglomerados no Brasil. Nas últimas duas décadas, as indústrias do setor de painéis reconstituídos de madeira têm empreendido grandes investimentos na implantação de novas unidades produtivas, além do aumento em novas áreas de plantios florestais, para assegurar o suprimento de madeira. Diante da demanda crescente pela madeira de reflorestamento na constituição dos mais diversos tipos de chapas de madeira, combinada com a necessidade de suprimento cada vez maior por esses tipos de produtos, surge também a necessidade de emprego específico da madeira de espécies alternativas, assim como o aproveitamento de resíduos dessas mesmas espécies (Iwakiri et al., 2012).

Uma das espécies mais promissoras para reflorestamento é a Acacia mangium Willd, da família Fabaceae, subfamília Mimosoidae, que ocorre naturalmente na região costeira da Austrália, na Indonésia, nas Ilhas Molucas e Java e na Província de Papua Nova Guiné, entre as latitudes de $1^{\circ}$ a $10^{\circ} \mathrm{S}$ e altitudes que vão desde o nível do mar até $800 \mathrm{~m}$ (Silva et al., 1996). Sua madeira é utilizada para produção de lenha, construções, móveis, aglomerados, papel e celulose, carvão, dentre outros.

Uma das etapas dos tratos silviculturais no cultivo da Acacia mangiun é a realização de podas, para retirada de galhos que possam, no futuro, desvalorizar a madeira para fins tecnológicos mais nobres, devido a presença dos nós, que podem ser fontes de redução de resistência mecânica, dificultar a trabalhabilidade, impedir uma adesividade adequada, dentre outros problemas. Essa prática acaba gerando um resíduo no meio florestal, que muitas vezes é descartado no ambiente ou queimado, causando prejuízo econômico e ambiental (Tuomela et al., 1996).

Esse resíduo florestal apresenta pequeno diâmetro, não sendo possível seu aproveitamento pelas indústrias de base tecnológica de madeira, tais como serraria e laminadoras. Assim, a agregação de valor a esse resíduo é de suma importância tanto do ponto de vista econômico quanto ambiental. Uma das possíveis aplicações seria utilizá-lo como matéria-prima para produção de aglomerados convencionais.

Nesse sentido, o objetivo desse trabalho foi avaliar a qualidade dos painéis produzidos a partir da madeira de desrama de Acacia mangium.

\section{Material e métodos}

A madeira de desrama, das árvores de Acacia mangium com 48 meses e DAP médio de $13,6 \mathrm{~cm}$, foi coletada na fazenda Impoeira, empresa Bee happy, localizada no Município de Curimatá na região sul do Piauí, sob as coordenadas $9^{\circ} 37^{\prime} 28^{\prime \prime} \mathrm{S} 44^{\circ} 07^{\prime} 12^{\prime \prime} \mathrm{W}$ e altitude de $650 \mathrm{~m}$. Para comparação, foram coletadas madeiras para fabricação de Pinus oocarpa e Eucalyptus grandis. As árvores de $P$. oocarpa e E. grandis foram abatidas no campus da Universidade Federal de Lavras (UFLA), Lavras, MG. Essas apresentavam, aproximadamente, 84 meses de idade. Os materiais foram encaminhados à Unidade Experimental de Produção de Painéis de Madeira (UEPAM) da UFLA para produção e análise das propriedades dos aglomerados convencionais.

$\mathrm{O}$ adesivo utilizado foi a uréia-formaldeído (UF), com teor de sólidos de $64,07 \%, \mathrm{pH}$ de 8,48 , viscosidade de $480 \mathrm{cP}$ e tempo de gelatinização de 53 segundos. A aplicação de adesivo foi de $8 \%$ em relação à massa seca das partículas. Da mesma forma, foi aplicada a emulsão de parafina a $1 \%$, em relação à massa seca das partículas.

As partículas "sliver" foram obtidas a partir do processamento de toretes em moinho de martelo. Após a geração, as mesmas foram peneiradas, sendo utilizadas para produção do painel aquelas partículas que ficaram retidas entre as peneiras de 10 e 30 mesh, o que somou $50,40 \%$ em relação ao total de partículas geradas. 
Posteriormente, foram secas até o conteúdo de umidade de $3 \%$ na base seca.

Na sequência, as partículas foram misturadas em uma encoladeira do tipo tambor giratório, sendo realizada a aplicação do adesivo UF e da emulsão de parafina. O colchão de partículas foi então prensado à temperatura de $160{ }^{\circ} \mathrm{C}$ e pressão específica de $3,92 \mathrm{MPa}$, por um período de 8 min, obtendo-se assim os painéis homogêneos.

Foram produzidos 6 painéis para cada material utilizado, cujas dimensões nominais e a densidade foram, respectivamente, de $480 \mathrm{~mm} \times 480 \mathrm{~mm} \times 15 \mathrm{~mm}$ e $700 \mathrm{~kg} \mathrm{~m}^{-3}$.

Retiraram-se 4 corpos de prova por painel, para avaliação das propriedades de flexão estática - DIN 52362 (Deutsches Institut Fur Normung, 1982); 6 corpos de prova por painel, para tração perpendicular - ASTM D1037 (American Society for Testing and Materials, 2006) e 2 corpos de prova por painel, para absorção de água e inchamento em espessura após $2 \mathrm{~h}$ e $24 \mathrm{~h}$ de imersão - ASTM D1037 (American Society for Testing and Materials, 2006). Os mesmos foram climatizados à temperatura de $20 \pm 3{ }^{\circ} \mathrm{C}$ e umidade relativa de $65 \pm$ $5 \%$. Foi utilizado o esquema de retirada de corpos de descrito por Mendes (2001).

Utilizou-se o delineamento inteiramente casualizado, com 6 repetições, em que os tratamentos foram considerados as diferentes espécies utilizadas. Utilizouse o teste de médias de Scott-Knott (1974), em 5\% de probabilidade de erro.

\section{Resultados e discussão}

Na Tabela 1 são apresentados os valores médios de densidade dos painéis, absorção e inchamento em espessura após $2 \mathrm{~h}$ e $24 \mathrm{~h}$ de imersão em água. Para densidade verifica-se que não houve diferenças estatísticas significativa entre os painéis estudados.
Os valores de densidade foram inferiores ao nominal $\left(0,70 \mathrm{~kg} \mathrm{~cm}^{-3}\right)$. Isso pode ser atribuído à especificidade das condições laboratoriais em relação ao processo industrial, com perdas de materiais durante o manuseio das partículas nas etapas de aplicação de adesivo, formação do colchão e prensagem dos painéis. De acordo com a norma CS 236-66 (Commercial Standard, 1968) todos os painéis produzidos foram classificados como de média densidade.

Para absorção de água, após 2 h e 24 h de imersão, observou-se destaque para os painéis produzidos com madeira de desrama de Acacia mangium e Pinus oocarpa, por apresentarem valores inferiores em comparação com madeira de Eucalyptus grandis. Possivelmente este fenômeno ocorreu devido a menor densidade da madeira de Pinus oocarpa e desrama de Acacia mangium. Assim, para se obter a mesma densidade nominal do painel é necessário um maior volume de partículas, as quais proporcionam maior razão de compactação e consequentemente menor quantidade de espaços vazios. Esta menor porosidade, segundo Vital et al., (1974) impede a penetração e retenção da água.

Em pesquisa realizada por Iwakiri et al. (2001), para painéis de madeira aglomerada com cinco espécies de pinus tropicais, os valores médios obtidos foram na faixa de $68,82 \%$ a $83,60 \%$, para absorção de água após $2 \mathrm{~h}$ e de $78,69 \%$ a 93,81\%, para absorção de água após 24 h. Os resultados apresentados para painéis produzidos com espécies de Pinus elliottii e Eucalyptus dunnii mostraram valores médios de absorção de água que variaram na faixa de $42,09 \%$ a $71,75 \%$, para $2 \mathrm{~h}$ de imersão e de 73,12\% a 80,05\%, para 24 h (Iwakiri et al., 1996). Dessa forma, os valores obtidos nesse trabalho com os painéis de Pinus oocarpa e desrama de Acacia mangium mostraram melhor desempenho, ou seja, maior resistência à umidade.

Tabela 1. Propriedades físicas dos painéis aglomerados para os diferentes tratamentos

\begin{tabular}{lccccc}
\hline \multicolumn{1}{c}{ Espécies } & $\begin{array}{c}\text { Densidade } \\
\text { painel (kg cm } \mathbf{3})\end{array}$ & $\begin{array}{c}\text { Absorção 2 h } \\
\mathbf{( \% )}\end{array}$ & $\begin{array}{c}\text { Absorção 24 h } \\
\mathbf{( \% )}\end{array}$ & $\begin{array}{c}\text { Inchamento 2 h } \\
(\mathbf{\%})\end{array}$ & Inchamento 24 h (\%) \\
\hline Desrama de Acacia mangium & $0,68 \mathrm{~A}$ & $27,18 \mathrm{~A}$ & $63,89 \mathrm{~A}$ & $9,90 \mathrm{~A}$ & $19,79 \mathrm{~A}$ \\
Pinus oocarpa & $0,66 \mathrm{~A}$ & $32,67 \mathrm{~A}$ & $63,43 \mathrm{~A}$ & $18,44 \mathrm{~B}$ & $39,51 \mathrm{~B}$ \\
Eucalyptus grandis & $0,65 \mathrm{~A}$ & $65,49 \mathrm{~B}$ & $90,16 \mathrm{~B}$ & $19,79 \mathrm{~B}$ & $44,18 \mathrm{~B}$ \\
\hline CV (\%) & 6,63 & 29,49 & 12,51 & 17,61 & 17,10 \\
\hline
\end{tabular}

Médias seguidas de mesma letra na coluna não diferem estatisticamente entre si, pelo teste Scott-Knott, com 5\% de de probabilidade de erro; $\mathrm{CV}(\%)=$ coeficiente de variação. 
Para inchamento em espessura, após $2 \mathrm{~h}$ e $24 \mathrm{~h}$ de imersão, destacam-se os painéis produzidos com desrama de Acacia mangium, por apresentarem baixos valores em relação aos demais painéis estudados. Aliado a isto, os mesmos mostraram inchamentos inferiores aos observados por Iwakiri et al. (2001), encontrando para essa propriedade, após $2 \mathrm{~h}$ e $24 \mathrm{~h}$ de imersão, valores de $25,20 \%$ e $32,70 \%$, respectivamente. Da mesma forma, Iwakiri et al. (2000), trabalhando com espécies de eucalipto oriundas do aproveitamento de serrarias, observaram valores de inchamento de $12,38 \%$ a $30,60 \%$, após $2 \mathrm{~h}$ e de $23,50 \%$ a 38,81\%, após $24 \mathrm{~h}$.

Contudo a norma de comercialização CS 236-66 (Commercial Standard, 1968) exige valores para inchamento em espessura, após $24 \mathrm{~h}$ de imersão em água, de no máximo 35\% para painéis de média densidade. Nesse sentido, apenas a espécie Acacia mangium apresentou valores compatíveis ao proposto nessa normatização.

De maneira geral, pode-se afirmar que, do ponto de vista das propriedades físicas dos painéis aglomerados, a madeira de desrama de Acacia mangium apresenta um grande potencial de ser utilizada como matéria prima para produção de painés, principalmente quando comparado a espécies tradicionalmente utilizadas pelo mercado.

Na Tabela 2 são apresentados os valores médios de flexão estática, em que foram avaliados os módulos de elasticidade (MOE) e de ruptura (MOR), e tração perpendicular dos painéis aglomerados das espécie de Acacia mangium, Pinus oocarpa e Eucalyptus grandis.

Para o MOE nota-se que o maior valor observado foi para Eucalyptus grandis $(2559,68 \mathrm{MPa})$, enquanto os painéis de Pinus oocarpa e de madeira de desrama de Acacia mangium apresentaram valores iguais estatisticamente entre si e inferiores ao obtido para os painéis de Eucalytpus grandis.

Tabela 2. Propriedades mecânicas dos painéis aglomerados.

\begin{tabular}{lccc}
\hline \multicolumn{1}{c}{ Espécie } & $\begin{array}{c}\text { MOE } \\
\text { (MPa) }\end{array}$ & $\begin{array}{c}\text { MOR } \\
\text { (MPa) }\end{array}$ & $\begin{array}{c}\text { Tração } \\
\text { perpendicular } \\
\text { (MPa) }\end{array}$ \\
\hline $\begin{array}{l}\text { Desrama de Acacia } \\
\text { mangium }\end{array}$ & $1803,78 \mathrm{~A}$ & $11,15 \mathrm{~A}$ & $0,59 \mathrm{~B}$ \\
$\begin{array}{l}\text { Pinus oocarpa } \\
\text { Eucalyptus grandis }\end{array}$ & 1830,96 A & $11,76 \mathrm{~A}$ & $0,27 \mathrm{~A}$ \\
\hline $\mathrm{CV}(\%)$ & $2559,68 \mathrm{~B}$ & $18,17 \mathrm{~B}$ & $0,73 \mathrm{~B}$ \\
\hline
\end{tabular}

$\mathrm{MOE}=$ módulo de elasticidade. $\mathrm{MOE}=$ módulo de ruptura. Médias seguidas de mesma letra na coluna não diferem estatisticamente entre si, pelo teste Scott-Knott, com 5\% de de probabilidade de erro. $\mathrm{CV}(\%)=$ coeficiente de variação.
Os resultados obtidos nesse estudo foram superiores aos apresentados por Naumann et al. (2008) para painéis de Eucalyptus urophylla e Schizolobium amazonicum, com valores médios, respectivamente de $734 \mathrm{MPa}$ e $1.873 \mathrm{MPa}$. Também Pedrazzi et al. (2006) encontraram valores de MOE entre 1.014 MPa e $1.477 \mathrm{MPa}$, para tratamento com $8 \%$ de adesivo, utilizando serragem de madeira de eucalipto.

Apenas os painéis produzidos a partir da madeira de Eucalyptus grandis apresentaram valores para MOE acima das exigências da norma de comercialização americana CS 236-66 (Commercial Standard, 1968), a qual estabelece valores mínimos de 2.401,96 MPa para os painéis de média densidade.

Para MOR observa-se que houve destaque para os painéis produzidos com madeira de Eucalyptus grandis, com valores superiores aos demais. Entretanto, ressaltase que do ponto de vista de utilização, todos os painéis atenderam aos requisitos exigido pela norma CS 23666 (Commercial Standard, 1968) que estabele valores mínimos para essa propriedade de 10,98 MPa.

Em relação a outras pesquisas, os resultados encontrados no presente trabalho ficaram acima dos observados por Brito et al. (2006) trabalhando com chapas de madeira aglomerada de Pinus elliottii Engelm., utilizando partículas oriunda de madeira maciça e maravalha; observando valores de MOR variando entre 7,14 e 11,15 MPa. Já Iwakiri et al. (2004) estudando madeira de Grevillea robusta A. Cunn. Ex R. Br. para produção de aglomerados, encontraram valores de MOR próximos aos observados nesta pesquisa, com variação entre 10,10 e 17,35 MPa.

Os maiores valores médios para tração perpendicular apresentados pelos painéis de Acacia mangium e Eucalyptus grandis foram superiores aos encontrados por Pedrazzi et al. (2006), com utilização de $8 \%$ de adesivo uréia-formaldeído e densidade de $0,65 \mathrm{~g} \mathrm{~cm}^{-3}$ do painel, encontrando valores de 0,17 e 0,13 $\mathrm{MPa}$, com a utilização de resíduos de Eucalyptus saligna, gerados pela indústria de celulose. Da mesma forma, Colli et al. (2010) encontraram para painéis aglomerados produzidos com Schizolobium amazonicum valor médio de tração perpendicular de $0,22 \mathrm{MPa}$.

A norma de comercialização americana CS 236-66 (commercial Standard, 1968) para painéis de média densidade, com colagem à base de uréia-formaldeído, estabelece valores mínimos de 0,42 MPa. Nesse sentido, os painéis produzidos com madeira de desrama de Acacia mangium ou com Eucalyptus grandis atenderam a norma de comercialização. 


\section{Conclusões}

Em geral, a maderia de desrama de Acacia mangium apresenta potencial de ser utilizada na produção de painéis aglomerados, com a maioria de suas propriedades próximas a dos painéis de Pinus oocarpa.

As principais propriedades físicas e mecânicas os painéis de madeira de desrama de Acacia mangium atenderam as exigências da norma CS 236-66 (Commercial Standard, 1968), com exceção do MOE.

\section{Agradecimentos}

À Fundação de Amparo a Pesquisa do Estado do Piauí (FAPEPI) e à Universidade Federal de Lavras (UFLA).

\section{Referências}

AMERICAN SOCIETY FOR TESTING AND MATERIALS. Annual book of ASTM: D1037: standard methods of evaluating properties of wood-base fiber and particles materials. Philadelphia, 2006.

BRITO, E. O. et al. Chapas de madeira aglomerada utilizando partículas oriundas de madeira maciça e de maravalhas. Scientia Forestalis, Piracicaba, v.1, n. 72, p. 17-21, dez. 2006.

BUFALINO, L. Avaliação da permeabilidade e sua influencia sobre as propriedades físicas e de colagem em painéis aglomerados. 2010. 76 f. Dissertação (Mestrado) - Universidade Federal de Lavras, Lavras.

COLli, A.; VITAL, B. R.; CARNEIRO, A. C. O.; SILVA, J. C.; CARVALHO, A. N. M. L.; DELLA LUCIA, R. M. Properties of panels manufactured with wood particles of paricá (Schizolonium amazonicum Huber ex.Ducke) and coconut fibers. Revista Árvore, v. 34, p. 333-338, 2010.

COMMERCIAL STANDARD. Mat formed wood particle board: CS 236-66. Wallingford, 1968.

DEUTSCHES INSTITUT FUR NORMUNG. Normen für Holzfaserplaten Spanplatten Sperrholz: testing of wood chipboards bending test, determination of bending strength: DIN 52362. Berlin, 1982. p. 39-40.

FAGUNDES, H. A. V. Diagnóstico da produção de madeira serrada e geração de resíduos do processamento de madeira de florestas plantadas no Rio Grande do Sul. 2003. 180 f. Dissertação (Mestrado em Engenharia Civil) - Universidade Federal do Rio Grande do Sul, Porto Alegre.

GUIMARÃES JUNIOR, J. B. et al. Painéis de madeira aglomerada de resíduos da laminação de diferentes procedências de Eucalyptus grandis, Eucalyptus saligna e Eucalyptus cloeziana. Revista Cerne, Lavras, MG, v. 17, n. 4, p. 443-452, out./dez. 2011.
IWAKIRI, S. et al. Produção de chapas de partículas de madeira de Pinus elliottii e Eucalyptus dunnii. Agrárias, Curitiba, v. 15, p. 34-41, 1996.

IWAKIRI, S. et al. Resíduos de serrarias na produção de painéis de madeira aglomerada de eucalipto. Scientia Agraria, Curitiba, v. 1, n. $1 / 2$, p. $23-28,2000$.

IWAKIRI, S. et al. Produção de chapas de madeira aglomerada de cinco espécies de pinus tropicais. Floresta e Ambiente, Seropédica, v. 8, n. 1, p. 137-142, jan./dez. 2001.

IWAKIRI, S.; SHIMIZU, J.; SILVA, J C.; MENESSI, C. H. S. D.; PUEHRINGHER, A.; VENSON, I.; LARROCA, C. Produção de painéis de madeira aglomerada de Grevillea robusta A. Cunn. ex R. Br. Revista Árvore, v. 28, n. 6, p. 883-887, 2004.

IWAKIRI, S. et al. Avaliação das propriedades de painéis aglomerados produzidos com resíduos de serrarias de nove espécies de madeiras tropicais da Amazônia. Acta Amazonica, v.2, n. 1, p. 59-64, 2012.

MENDES, L. M. Pinus spp. na Produção de painéis de partículas orientadas (OSB). 2001. 156 f. Tese (Doutorado em Tecnologia e Utilização de Produtos Florestais) - Universidade Federal do Paraná, Curitiba.

MOSLEMI, A. A. Particleboard. Carbondale: Southern Illinois University, 1974.

NAUMANN, R .B.; VITAL, B. R.; CARNEIRO, A. C. O.; LUCIA, R. M. D.; SILVA, J. C.; CARVALHO, A. M. M. L.; COLLI, A. Propriedades de chapas fabricadas com partículas de madeira de Eucalyptus urophylla S. T. Blake e de Schizolobium parahyba Herb. Árvore, Viçosa, MG, v. 32, n. 6, p. 1143-1150, 2008.

PEDRAZZ I, C. et al. Qualidade de chapas de partículas de madeira aglomerada fabricadas com resíduos de uma indústria de celulose. Ciências Florestais, Santa Maria, RS, v. 16, n. 2, p. 201-212, 2006.

SCOTT, A.; KNOTT, M. Cluster-analysis method for grouping means in analysis of variance. Biometrics, Washington D.C.,v. 30, n. 3, p. 507-512, 1974.

SILVA, F. P.; BORGES, R. C. G.; PIRES, I. E. Avaliação de procedências de Acacia mangium Willd, aos 63 meses de idade no Vale do Rio Doce. Revista Árvore, Viçosa, MG, v. 20, n. 3, p. 267-278, 1996.

TSOUMIS, G. Science and technology of wood: structure, properties, utilization. New York: Chapman \& Hall, 1991. 494 p.

TUOMELA, K.; OTSAMO, A.; KUUSIPALO, J.; VUOKKO, R.; NIKLES, G. Effect of provenance variation and singling and pruning on early growth of Acacia mangium Willd. plantation on Imperata cylindrica (L.) Beauv. dominated grassland. Forest Ecology and Management, v. 84, p. 241-249, 1996.

VITAL, B. R., LEHMANN, W. F. Y BOONE, R. S. How species and board densities affect properties of exotic hardwood particleboards. Forest Products Journal, Madison, v. 24, n. 12, p. 37-45, 1974. 
